\title{
7 Weak Links and the Stability of Organisms
}

With this part of our trip, we discover more familiar parts of Netland. This chapter will be about ourselves. Our body is a network of cells and organs. Unfortunately, this complex world is largely unexplored from the standpoint of network behavior. This is partly because elements and modules are mixed up here. Sometimes an organ may be regarded as an element of the body net, whilst in other cases it has to be treated as a module of its cells. Moreover, the complexity of the interactions makes exploration and description even more difficult. This is why I will sometimes use examples from the animal world to bring out the network properties of this exciting network: our own body.

\subsection{Immunological Networks}

The immune system has to solve four problems:

- the self/non-self recognition problem,

- the signal-to-noise problem,

- the context problem,

- the response problem. ${ }^{1}$

This complex task can only be solved by a network (Cohen 1992a). Immune cells provide perhaps the most sophisticated network in our body. Not only is each cell different from any other and capable of interacting with any other, but the system topology changes continuously and long-range interactions are also possible via cytokines and other mediators. This system offers perhaps the largest repertoire of events which call for stabilization. The idea of immunological networks was first introduced by Niels K. Jerne as the idiotype network, i.e., an

\footnotetext{
${ }^{1}$ These four basic problems of the immune system can be formulated with the following three questions: For the first two, what should the immune system attack? For the third, when should the immune system attack? For the fourth, how should the immune system attack?
} 
inter-reactive network, where antibodies of one lymphocyte serve as an antigen for another ${ }^{2}$ (Jerne, 1974; 1984). Later this concept was extended to networks of natural autoantibodies, which has been called the immunological homunculus, or immunculus by Irun Cohen (1992a; 1992b). Cytokines and other participants of the immune response also form networks.

Immunological networks possess all the properties forming the signature of a network presented in Chap. 2. The reactivity pattern of the repertoires of both $\mathrm{T}$ and $\mathrm{B}$ lymphocytes, which denotes a set of positive and negative actions on a series of antigens, has a scale-free distribution (Burgos, 1996). The scale-free pattern invokes the selfsimilarity of fractal structures. Indeed, Jerne (1984) noted that the immune system is complete in the sense that, if we were able to remove $90 \%$ of B-cell-derived antibodies randomly, the remaining ones would still represent a complete repertoire.

Among the major network properties, the highly flexible modularity of the immune network seems to be especially important. Modularity helps the dissection of the network of natural autoantibodies, the immunculus, from the networks of acquired immune response to various antigens. Modularity also gives rise to stabilized, localized immune activation circuits as well as to immunological memory (Cohen and Young, 1991; Varela and Coutinho, 1991; Weisbuch et al., 1990). The segregation of a module within the immune network is similar to the segregation of synchronized oscillators within a larger network described by Winfree (1967) and the behavior of the Boolean networks of Kauffman (1969). Utilizing the generality of the immune response, distortions of the immune network have been proposed as highly sensitive potential markers of pathogenic metabolic changes (Poletaev and Osipenko, 2003).

The dynamical properties of the immune network start from its very development. The development of B cell clones in newborns leads to a general decrease in the connectivity of network members from several dozen to around 6 to 9 . Low connectivity comes in parallel with high affinity interactions and massive antibody production (De Boer and Perelson, 1991). In agreement with these changes, the B cell repertoire becomes more restricted as mice grow from 1 to 2 weeks old (Burgos, 1996). These changes are reminiscent of the random to scale-free topological phase transition described in Sect. 3.4. In that transition, the highly connected, low complexity network was transformed to a more

\footnotetext{
${ }^{2}$ The sequence of the idiotype network antibodies may be continued: the antibodies of the second lymphocyte serve as antigen for the third, etc.
} 
sparsely connected, but complex network. The change was provoked by a decrease in the available resources.

During these changes, the immune network can recruit and lose elements, change topology, and show restricted percolation phenomena (Brede and Behn, 2002; DeBoer and Perelson, 1991). The connection density has a profound effect on immune properties. If there are too few connections, the network cannot function. This seems rather obvious. However, if there are too many connections, the network will not function properly either (DeBoer and Perelson, 1991; Varela et al., 1991). This resembles the dilution effect which occurs when a node has too many neighbors and their influence is diminished. As we saw in Sect. 3.3, the dilution effect gives rise to unpredictable cascading failures (Watts, 2002). The immune network displays scale-free behavior in time, with $1 / f$ noise-type fluctuations ${ }^{3}$ in the idiotypic network of natural antibodies (Lundkvist et al., 1989), which is disturbed in autoimmune disease (Varela et al., 1991).

Antigen exposure can be regarded as a disturbance of the original network status which provokes a relaxation phenomenon (Varela and Coutinho, 1991). This assumption makes it much easier to understand why the Watts condition (Watts, 2002) mentioned above may appear deleterious for the overconnected immune net. In the immunological network, relaxation is achieved by a massive change in the elements, connections and topology of the network as a whole. In agreement with this, the $\mathrm{B}$ cell repertoire becomes wider after antigen exposure (Burgos, 1996).

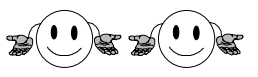

\section{Antigen hunger: A potential basis for immuno-}

logical diseases. Let us reconsider the above statements for a moment. If antigens provoke the relaxation of the immunological network (Varela and Coutinho, 1991), meaning that antigens serve as a resource for the immune net, and not long after birth the immunological network is likely to undergo a random to scale-free network transition, than in our early childhood our immunological network could be said to have indulged in a plethora of antigens. This should not be considered as a tragedy. Just as our brain feels well only when it is bombarded by information, our immune system feels well only if it is challenged by antigens. The young immune system is not fighting with the antigens of the neighboring world. In fact, quite the opposite. Repeated exposure to antigens keeps the young immune system perfectly resourceful and relaxed, allowing it to remain in the random phase, and prepared for

\footnotetext{
${ }^{3}$ This is the same as $1 / t$ noise, a form of pink noise (see Sect.2.1).
} 


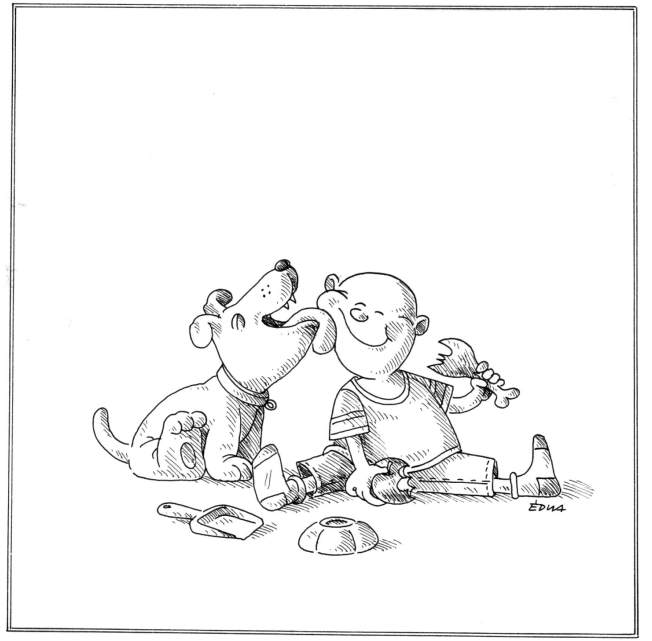

Fig. 7.1. Just as our brain feels well only if it is bombarded by information, our immune system feels well only if it is challenged by antigens

any future challenge and change. So the situation may actually be reversed. When we moved into the first caves, got our first clothes and grew up, our immune system was deprived of the joyful source of relaxation and an increasing antigen hunger developed. From this point of view, the increasing sterility of the current epoch is a true disaster, since it deprives the immune system even further of the much-awaited antigen relaxation. No wonder that increasing tensions develop in the immune system, which may lead to bursts of inflammation, allergy (Yazdanbakhsh et al., 2002), autoimmunity or immune deficiency. ${ }^{4}$ Many of these bursts may follow a scale-free pattern, resembling the avalanches of self-organized criticality described in Sect. 3.3. It is quite likely that we will soon have to administer regular 'dirt injections' of carefully selected antigens, to ensure the proper relaxation of our immune system. In a few years from now, this immune repair may be as natural a behavior as the other regular relaxation repair we indulge in, e.g., eating, sleeping, and laughter.

After the clone selection theory of Burnet (1959), the later network concepts of immune recognition (Jerne, 1974; 1984; Cohen 1992a) hy-

\footnotetext{
${ }^{4}$ In extreme cases, chronic and severe antigen deprivation may even produce a series of topological phase transitions leading eventually to a transition from the star phase to the isolated subgraph phase, which can in fact be identified with a total collapse of the immune system.
} 
pothesized a lot more weak links in the immune network. ${ }^{5}$ The degenerate pathways whereby various packages of antigens interact with the immune system and provoke an immune response certainly give rise to numerous weak links, as reflected in the models (Brede and Behn, 2002). In spite of this increasing knowledge of the immunological network, consideration of the role of weak links in the stabilization of the immune response are premature, since we do not yet have enough data to assess the effect of the affinity repertoire on the stability of an immune net containing millions of differing elements. However, as a final remark, let me demonstrate the strength of the concept with a hypothetical example.

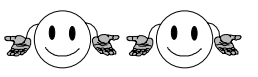

Autoimmune vaccination focuses and probably stabilizes the immune response. Vaccination is no longer an intervention used exclusively against infections. It is now becoming accepted as an efficient treatment against autoimmune diseases as well. Here vaccination modulates weak links by rearranging regulatory connections of the immunculus (Cohen $1992 \mathrm{~b} ; 2002)$. What happens if the vaccination not only redirects but also stabilizes the immune response by introducing additional weak links? "Something is wrong here. A direct immune response for a new antigen should introduce strong links if any, not weak ones." This treatment indeed introduces strong links in the form of the immune response against the newly added antigen. However, in comparison with this strong response, all the residual responses including the autoimmune responses become weak, even if they retain their original strength on an absolute scale. Acquiring the status of weak links, the former strong links of the autoimmune disease now contribute to the stabilization of the immune response. Having smaller fluctuations, the autoimmune response will not reach the activation threshold very often and the patient's symptoms will improve. We have a little data to support this model. The loss of scale-free characteristics of autoantibody fluctuations in autoimmune disease (Varela et al., 1991) may reflect a distortion in the topology and link strength distribution of the underlying immune network structure. ${ }^{6}$ In agreement with the model by Stewart et al. (1989), which suggested that autoimmunity develops due to an inadequate connectivity of self-reactive lymphocyte clones, vaccination may help to shift the immune network link strength pattern back to the scale-free distribution, where stabilizing weak links predominate.

\footnotetext{
${ }^{5}$ Jerne himself marked the third generation of anti-idiotypic antibodies as 'buffering sets' in his original publication in 1974.

${ }^{6}$ Self-organized networks tend to be scale-free in multiple dimensions (see Sect. 2.2 for more details).
} 


\subsection{Transport Systems}

Transport systems were introduced in Sect. 2.2, where I described the allometric scaling laws. These laws are empirical laws concerning the scale-free behavior of metabolic rates, heartbeats and lifespan, to name but a few contexts where they are relevant. All these measures behave as a linear function of the $1 / 4$ or $3 / 4$ power of the body mass. ${ }^{7}$ As described in Sect. 2.2, the 3/4 power law dependence of the metabolic rate is difficult to explain if one starts from simple geometrical considerations. One of the examples above, namely, the metabolism, depends on area. Mass obviously depends on volume, which would give a $2 / 3$ power law function rather than $3 / 4$. However, if the geometry of the blood vessels is taken into consideration, a $3 / 4$ power law function arises. The geometry of the branching transport network does indeed show a scale-free, fractal distribution both in our blood vessels and lungs (Banavar et al., 1999; McNamee, 1991; West et al., 1997). Allometric scaling can be extended to individual cells (West et al., 2002), and is in good agreement with the assumptions concerning the scale-free, fractal geometry of the cytoplasm (Aon et al., 2004b). Three-dimensional, scale-free networks display an optimized degree of efficiency, suggesting that evolution shaped them to minimize the costs of transportation (Garlaschelli et al., 2003).

The blood vessel network is degenerate. The branching network of vessels represents an arterial tree and is connected to a similar venous network. However, this tree is not regular. A small difference between vessel lengths and radii has to be introduced and can be experimentally verified to explain the observed heterogeneity of blood flow (van Beek et al., 1989). Collateral blood vessels do not duplicate the transport function. They perform the same function slightly differently. They are degenerate. Thinking back to Sect. 4.5, we may guess the type of links that arise when degeneracy occurs.

Once again, we find an interesting coexistence of scale-free behavior in space and time. Heartbeat dynamics in an undisturbed state

\footnotetext{
${ }^{7}$ The allometric scaling laws follow the equation $P=c M^{\alpha}$, where $P$ is the property, $c$ is a constant, $M$ is the mass of the organism or organelle, and $\alpha$ is a scaling exponent which depends on the nature of $P$. The most thoroughly studied of these is the basal metabolic rate, which obeys Kleiber's law (1932). Here $P$ is the basal metabolic rate, i.e., the amount of energy per unit time required by a living organism to remain alive, and the scaling exponent is $3 / 4$. The value of the exponent is different in the other examples. Hence, the dependence of heartbeat and lifespan have an $\alpha$ exponent of $-1 / 4$ and $+1 / 4$, respectively. As a word of caution, note that this simple rule is sometimes overinterpreted (Dodds et al., 2001).
} 
has multifractality, which means that the scale-free behavior can be described by more than one exponent. ${ }^{8}$ However, this is true only for a healthy person. In patients suffering from congestive heart failure, multifractality is lost and gives rise to much simpler scale-free behavior. This behavior can be characterized by a very narrow range of scale-free exponents and can be regarded as monofractal (Ivanov et al., 1999). The simplification of the pattern in a disease state resembles the loss of scale-free characteristics of autoantibody fluctuations in autoimmune disease (Varela et al., 1991), described in the last section.

\subsection{Muscle Net}

The stability of our movements requires a well-orchestrated interplay of various agonist and antagonist muscles and their governing neural functions. The generation of muscle force requires the dynamic recruitment of a large number of motor units with tens of thousands of individual neuronal commands. This process would certainly make the motor function very suitable for network analysis. However, the network approach is still largely absent from this field. As an example of the sporadic data indicating network behavior, Duarte and Zatsiorsky (2000) measured pressure fluctuations in experimental subjects standing on a force plate. The fluctuations during prolonged unconstrained standing showed a scale-free distribution.

Analyzing muscle control from the output, the movements and the generated force provide a good measure of system stability. The complexity of movements, as well as the variability of maximal forces and discharge speeds between motor units all make muscle control a noisy process. The behavior of noise becomes greater with advanced age (Enoka et al., 2003). The endpoint variability of the movement depends on the signal-to-noise ratio, which can be adjusted by the stiffness of the affected muscles (van Galen and Huygevoort, 2000). In other words, if we need higher precision, we flex our antagonistic muscles together with a greater force. Precision comes with struggle. (Many of my colleagues probably agree that more $\mathrm{PhD}$ students should take a course on muscle control to learn about this.)

\footnotetext{
${ }^{8}$ For a more detailed description of multifractality, see Sect. 2.2 or the glossary in Appendix B.
} 


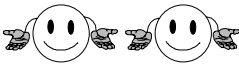

\section{An alternative explanation of struggle benefits.}

A balance of agonist and antagonist muscles at a higher force level invokes higher signals, which may increase the threshold and make the background noise less effective (van Galen and Huygevoort, 2000). However, another explanation may also be given. Yes, weak links again. Similarly to the idea outlined in Sect. 7.1, the muscle control system may use the increased strength of its key elements to make the strength distribution more uneven, and the stabilizing effect of weak links more pronounced. We should never forget: a weak interaction can be weak only in the presence of some really strong interactions. The strength increase may come only by the simultaneous flexing of antagonistic muscles, if we want to preserve the main character of the original movement. We probably need a struggle of opposing strengths to achieve stabilizing weakness.

The analysis of the output of muscle control already gave some hints about the potential involvement of network stability and weak links in the regulation of movement precision. However, an analysis of the input, the concerted activation of the numerous motor units, seems to be even more promising. Motor units are governed by the activation of motor neurons, which is often a synchronized process. However, the extent and level of synchronization are finely tuned in both the time and the frequency domains. Increased synchrony reduces the time required to reach maximal force (Semmler, 2002). However, the same increase induces larger fluctuations in muscle strength, decreasing the steadiness of the exerted force (Semmler et al., 2003; Yao et al., 2000). We have agreement with the output analysis above, since a smaller synchrony between motor units increases the chances that alternative, canceling forces will act against each other, which is the case for the increased muscle stiffness mentioned above. Table 7.1 summarizes a large number of examples to show that a decrease in motor unit synchrony leads to an increase in precision; and vice versa, a synchrony increase makes the final movement noisier.

From the exciting and highly coherent list of experimental data in the table, let me highlight some of the results of Semmler and Nordstrom (1998). They found that the synchrony of the index finger motor units increased as follows:

musician's right (dominant) hand = musician's left hand $=$ control person's right hand $<$ control person's left hand $=$ weight lifter's right hand $=$ weight lifter's left hand . 
Table 7.1. An intermediate level of motor unit synchronization is needed to achieve maximal movement precision

\begin{tabular}{|c|c|c|}
\hline Condition & Motor unit synchrony & References \\
\hline Aging & Coherence increases & Semmler et al., 2003 \\
\hline $\begin{array}{l}\text { Holding versus moving } \\
\text { of object by macaque } \\
\text { monkeys }\end{array}$ & $\begin{array}{l}\text { Increased synchrony } \\
\text { in motion }\end{array}$ & Baker et al., 2001 \\
\hline $\begin{array}{l}\text { Musicians versus } \\
\text { weight lifters }\end{array}$ & $\begin{array}{l}\text { Decreased synchrony } \\
\text { in skill training versus } \\
\text { strength training }\end{array}$ & $\begin{array}{l}\text { Semmler and Nordstrom, } \\
1998\end{array}$ \\
\hline $\begin{array}{l}\text { Skilled hand versus } \\
\text { other }\end{array}$ & $\begin{array}{l}\text { Decreased synchrony } \\
\text { of skilled hand }\end{array}$ & $\begin{array}{l}\text { Semmler and Nordstrom, } \\
1998\end{array}$ \\
\hline $\begin{array}{l}\text { Thumb and index finger } \\
\text { versus other fingers }\end{array}$ & $\begin{array}{l}\text { Decreased synchrony } \\
\text { of thumb and index } \\
\text { (skilled) fingers }\end{array}$ & Bremner et al., 1991 \\
\hline $\begin{array}{l}\text { Stroke patients versus } \\
\text { healthy subjects }\end{array}$ & $\begin{array}{l}\text { Increased long-term } \\
\text { synchrony of stroke } \\
\text { patients }\end{array}$ & Datta et al., 1991 \\
\hline $\begin{array}{l}\text { Parkinson's disease } \\
\text { patients versus } \\
\text { healthy subjects }\end{array}$ & $\begin{array}{l}\text { Increased long-term } \\
\text { synchrony in } \\
\text { Parkinsonism }\end{array}$ & Datta et al., 1991 \\
\hline
\end{tabular}

Thus, in terms of precision and motor unit synchrony, piano or flute training gives you two right hands instead of one, whereas weight lifting leaves you with two left hands. In contrast to the struggle mentioned above, brute force is not really helpful to achieve precision. Here is another lesson for $\mathrm{PhD}$ students!

The need for increased muscle sync: birth. Motor unit synchronization and the subsequent destabilization of the muscle net can be helpful. And not just helpful, but essential. Childbirth requires the contraction of the uterus. Approaching term, the incoherent contractions become more and more synchronized leading to an increased susceptibility of the fetus-mother system to external perturbations (Sornette, 2002). This may actually work like an integrative computer so that, when the increasingly synchronized contractions exceed a threshold with the addition of the integrated perturbations, the baby is born. 
In summary, if we have a predictable task, requiring a well-practised, fast action, like weight lifting, a large synchronization develops. (From this point of view, birth may be regarded as a special form of weight lifting.) This is achieved by strong links between muscle units. On the other hand, if we have an unpredictable, complex task requiring movement precision, like music performance, we need a submaximal, optimal level of motor unit synchronization. Partial synchronization, which causes a divergence in the action of individual motor units, is a typical case of weak link generation at the muscle network level. ${ }^{9}$ Strong links are required for well-defined, predictable tasks, which already have an adaptive response. Weak links are necessary for changing and carrying out unknown tasks of high complexity. I return to the more general consequences of these statements in Sect. 9.5, where I redefine the differences between engineers and evolution as a tinkerer. Engineering usually solves a well-defined, predictable task, in contrast to evolutionary tinkering, which solves unpredictable, changing tasks of high complexity.

The emergence of weak links at least coincides with the stabilization of our movements. Where can we find a causative link? Movement of muscle units is governed by motor neurons residing in the central nervous system. Thus the final source of synchronization and network regulation is a level higher. This level, the network of neurons and associated glial cells, will be the subject of the next section.

\subsection{The Neuro-Glial Network}

The maximal information processing capacity of the human cortex is around a terabit per second, which is comparable to the backbone capacity of the world Internet in 2002. The human cerebral cortex contains approximately 8.3 billion neurons and 67 trillion connections. The length of these connections in a single human brain is between 8 and 800 times the diameter of the Earth. It is not therefore surprising that cortical neurons form a rather complex network. But since information processing is very costly, ${ }^{10}$ it is no more surprising to find that

\footnotetext{
${ }^{9}$ Once technical advances permit, this important lesson may be applied in the construction of artificial limbs.

${ }^{10}$ Besides skeletal muscle our brain is the other major energy consumer in our body: just try to sit calmly and read this book without eating for several hours and you will see how hungry you become - not for more science in this case, but for food.
} 
only about 1 to $16 \%$ of neurons operate at any given time (Aiello and Wheeler, 1995; Laughlin and Sejnowski, 2003; Sporns, 2003).

Our brains seem to have read Chap. 2 rather well, learning the lesson and displaying all the usual features of a typical network. The neural net is a small world which is ensured by dense local connectivity and relatively few long-range connections. In agreement with this, neural connection length displays a scale-free distribution. The neural network is modular and the modules are highly interconnected. At intermodular boundaries, special overlaps, the so-called fringe areas, can be found, which may lead to either facilitation or occlusion of the neighboring neuronal modules. Finally, the neural net is nested. This means that the network of neurons contains local circuits, which are multiple synaptic junctions with complex electronic and chemical interactions. These local circuits further contain a number of molecular networks in the individual synapses. All these features serve a compromise between maximization of computational power and complexity and minimization of the costly wiring and maintenance needed to achieve this (Agnati et al., 2004; Buzsáki et al., 2004; Eguiluz et al., 2005; Kniffki et al., 1993; Laughlin and Sejnowski, 2003; Sporns, 2003).

Unless you are familiar with neuro-anatomy, or you are involved in one of the rapidly developing neuronal imaging techniques (Freund, 2003; Gulyas, 2001), it will be rather difficult to obtain a direct view on the network properties of the neural net above. However, there is a related feature which you definitely will have observed. In agreement with the above structure, our mistakes also follow scale-free statistics (Gilden et al., 1995). This means that when we try to make a replica of a target length in space or time, most of the time we make only a small error. However, we sometimes definitely make a bigger mistake. Is this bad news? It will become even worse. Sooner or later we will make a real blunder, too. (In another way, this is also good news: I have at least one excuse for a big mistake in this book.) Scale-free statistics is characteristic of error in a large variety of cognitive $\operatorname{tasks}^{11}$ (Gilden, 2001).

Higher brain functions emerge from a transient synchrony between neural cells. Synchronization has already been postulated by Berstein in his classic paper of 1945, where he stated that (Sporns and Edelman, 1998): "There has to be an inevitable synchronization, both by frequency and by phase of numerous low-frequency oscillators in the

${ }^{11}$ The scale-free error distribution is characteristic of response latency, repetition and discrimination accuracy. 
cortex." Indeed, it has been demonstrated that temporarily synchronized neurons ensure successful memory formation (see Sect. 3.5) (Fell et al., 2001). Neural networks demonstrate several oscillatory bands between $0.05 \mathrm{~Hz}$ and $500 \mathrm{~Hz}$. Slow oscillations require very large networks and efficiently modulate faster oscillations confined to smaller neuronal space (Buzsaki and Draguhn, 2004). Recent data showed the existence of two dynamic, diametrically opposed, widely distributed and anticorrelated networks of brain oscillators (Fox et al., 2005). In a number of diseases, such as Alzheimer's disease, autism, schizophrenia and attention-deficit disorder, a disturbed neural synchrony was observed (Stam et al., 2005; Stelt et al., 2004).

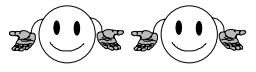

Saints and geniuses: Exceptional cases of extensive synchronization and coherence. Extensive synchronization and coherence of electroencephalograms (EEG) reflect an increase in long-range connectivity of the neuronal network. Rather interestingly these states were found to be characteristic of emotionally intensive states, tasks requiring efficient information processing or creativity, giftedness and meditation (Aftanas and Golocheikine, 2001; Jausovec and Jausovec, 2000; Orme-Johnson and Waynes, 1981; Petsche, 1996). (I just note in parentheses that the nested sync I suggested as a possible explanation of the Jungian synchronies of fulfilled prayers and dreams also invokes similar mental states.)

As described in Sect. 3.5, synchronization is helped by small-worldness and scale-freeness, typical features of neural networks. Weak coupling has also been suggested to facilitate synchronization (Sect. 3.5). What are the weak links between neurons? Many of the indirect neural regulatory mechanisms (Paton and Vizi, 1969; Vizi, 1979; 1984) and the so-called volume transmission (Agnati and Fuxe, 2000; Agnati et al., 1986) of diffusible messengers, such as nitric oxide, or carbon monoxide, almost certainly play a role in accomplishing this important task. However, we do not know enough about the strength or the sophisticated regulation of these interactions to assess their contribution in a general manner.

Besides direct neural transmission, or volume transmission, astrocytes provide another candidate for weak interneural links. ${ }^{12}$ Astrocytes demarcate gray matter regions from gliovascular units (Nedergaard et al., 2003) and could easily be one of the weak linkers between the modules of the neural net. The ratio of astrocytes to neurons in-

\footnotetext{
${ }^{12}$ I am grateful to Péter Száraz for this idea.
} 
creases steadily as brain function develops. They form an electrical syncytium (Nedegaard et al., 2003) which ensures a weak link with neighboring neurons. Astrocytes have coordinated calcium signals with neighboring neurons and regulate synaptic transmission, synaptogenesis, synaptic maturation and elimination (Hirase et al., 2004; Newman, 2003d; Slezak and Pfrieger, 2003), which predispose them as an ideal candidate for a stabilizing weak link in neural networks.

We may have quite an elaborate network of weak links in our brain. These links may help synchronization and the integration of brain function. What are the consequences of their contributions? Dreams, learning and even our consciousness may all require weak links in the neural net. These complex brain functions lead us to human behavior, which will be the subject of the next section after the following comments on dreams, learning and various levels of consciousness.

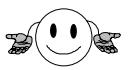

Weak links may help us to dream. The longest and most intense dreams occur during the rapid eye movement (REM) phase of sleep and can be characterized by (a) memory selection, (b) bizarre and unrecognizable representation of memories, and (c) high emotional content. Cognitive tests on associative memory suggest that REM dreams are modulated primarily by weak neocortical associations (Stickgold et al., 2001).

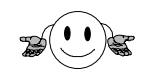

Weak links may help learning. The aim of learning is to make strong links in neural networks. However, most of our memory is helped by associations with weakly defined subsystems like colors, emotions, smells, etc. This is called the context effect. If divers learn words under water, they remember them much better under water than on the shore. If we learn words under the influence of alcohol, we will remember them better if we drink again. Similarly, alcoholics find hidden reserves of money or liquor when they get intoxicated again (Goodwin et al., 1969; Smith and Vela, 2001). Recent data indicate that the slow-wave, non-REM phases of sleep may be involved in remodeling of the neural network, thus assisting the consolidation of a previous learning process (Huber et al., 2004). It is currently unknown how this network remodeling affects weak versus strong links between neurons and neuronal modules.

Weak links and consciousness. Consciousness facilitates widespread connections between otherwise independent modules of the neural net increasing both the complexity and synchronization of brain function. 
Both the loss of neural synchronization or over-synchronization, e.g., in slowwave sleep or in epileptic seizures, respectively, reduce complexity and may lead to unconsciousness. The simultaneous presence of functional integration and segregation, parallel and recursive signaling (called reentry), as well as the large repertoire of differentiated neural states are key features of neural complexity and the conscious state. These all require an optimal amount of ever-changing synchronization (Baars, 2002; Tononi and Edelman, 1998; Tononi et al., 1992; 1998). Synchronization and connection of divergent brain areas and functions are all helped by weak links.

(1) Reflections of the outside world. We have quite numerous mechanisms in which we either construct internal images of the outside world or allow the triggering of pre-set responses by the outside world. Internal images of the outside world are formed when we imagine other people's mental state by adopting their perspective with the help of a special set of neurons called mirror neurons (Gallese and Goldman, 1998). Triggering of pre-set responses by the outside world occurs when we become exposed to unconscious information like primes, which are images too short to be recognized consciously (Kunde et al., 2003). In all these mechanisms, a broad coherence of the neuronal network is expected to operate. This coherence also requires a broad synchronization and connection of divergent brain areas, which is once again helped by weak links. These weak links not only allow the various levels of our consciousness to develop, but also lead us to the next section: the human psyche.

\subsection{Psycho Net}

The network properties of our brain invoke a network approach to rationalize and understand the human psyche. The Freudian revolution broke the transparency of the 'Cartesian theatre' of our inner world, showing that it cannot be discovered even by its owner (Pleh, 1988). This broken world was calling for a more complex approach. The basis of psycho nets was established by Carl Gustav Jung in 1921, when he published his landmark book on psychological types. Stern's differential psychology (Stern, 1911), typology (Jung, 1969; Kretschmer, 1921) and factor analysis (Cattell 1978; Eysenck, 1970; Spearman, 1931) are some of the major schools and methods of psychology which are inherently related to network studies.

In spite of all these psychological networks, rather surprisingly, neither the four major network properties mentioned in Chap. 2, small- 
worldness, scale-freeness, nestedness and weak-linkness, nor the dynamic network characteristics such as the presence of a giant component, percolation, self-organized criticality, topological phase transitions, synchronization, etc., have been systematically addressed in order to understand the structure and dynamism of our psychology. In the rest of this chapter, I outline a few starting ideas to illustrate the wealth of potential applications of the network paradigm in psychological studies.

As one of the rare examples of the network-related dynamical aspects of psychological events, I have already mentioned the scale-free statistics of ticks in Tourette-syndrome patients (Sect. 3.2) (Peterson and Leckman, 1998). The variation of the time interval between two ticks can be interpreted as a self-organized criticality event, a tick quake. During a tick quake a gradual tension develops, and the relaxation comes in the form of the tick.

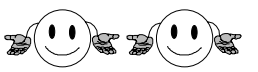

Adolescence as a psycho quake. The development of the psyche is not monotonic. Evolutionary periods are interspersed with sudden bursts of development, as in the punctuated equilibrium of evolution (Gould and Eldredge, 1993). One of these bursts occurs in adolescence. Here self-accomplishment and partner contacts are often felt to be delayed and a tension gradually develops. On the other hand, the teenager leaves the safe circles of the family and school and becomes increasingly exposed to completely different environments, where she has to cope with the new situations alone. From time to time, these occasions may trigger an avalanche-type development of the psyche, or psycho quake.

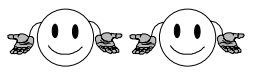

A healthy psyche needs a balance of strong and weak links. We need a healthy balance of strong and weak links to maintain our psychological stability. Unbalanced links characterize a number of psychic disorders:

- Monomaniacs and multi-talented people. Both monomaniacs and multitalented people tend to be emotionally unstable. Monomaniacs may have an emotional network resembling a star network, where all the links are strong. On the other hand, multi-talented people are closer to an all-weak, random network. To help their stabilization, monomaniacs require various secondary activities, while multi-talented people need strong guidance, to provide their missing weak or strong links, respectively.

- Autism. Another potential example of a strong-link surplus to various, and not necessarily personal elements of the environment may be autism, 
where novel situations, ambiguity, and the appearance of weak links may cause a panic reaction revealing a high level of instability.

- Emotional traumas. Emotional traumas often result in a closed personality, where the former personal network is reduced to a few strong links.

- Borderline personality disorder. Undeveloped, broken or unbalanced emotional links of early childhood may cause a borderline personality disorder, where the link imbalance is extended during adolescence and leads to a general instability of the personality.

Linville (1987) showed that the complexity of the self protects against the destabilizing effects of stress. A higher self-complexity here may mean multiple social dimensions or friends from a grossly different social context. All of these result in an increased number of weak links between the various selfaspects and cognitive modules, which counteract the destabilizing effects of stress. A balance between strong and weak links is also a prerequisite of the 'flow', a state of harmonious creativity (Csikszentmihalyi, 1990). ${ }^{13}$

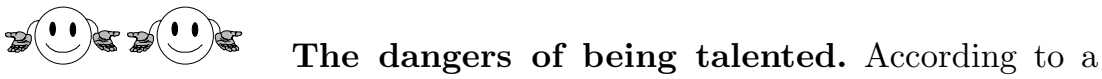
recent report by Beilock and Carr (2005), individuals with a high working memory capacity showed a decrease in capacity for accurately solving difficult mathematical problems when they were in a situation leading to high emotional pressure. ${ }^{14}$ So far the results are rather obvious: stress provokes an attention deficit. The interesting part was that individuals with a low working memory capacity showed a much smaller decrease under pressure. In fact, the talented group lost all its advantage in the stressful situation. As an explanation for these observations, the authors suggest that performance pressure may consume the working memory capacity surplus of talented individuals. The network approach may provide an alternative explanation. As described in Chap. 6, extreme abilities may correlate with a generally more unbalanced status of the underlying cellular networks. If the talented person is already less well balanced from the beginning, the larger intrinsic noise may provoke a stochastic resonance and may help the stress to exceed an 'inability threshold'. In the average person, the intrinsic noise is small, and stress thus has no chance of reaching this inability threshold.

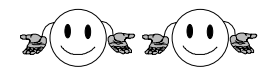

STRONGLINKERS and WEAKLINKERS: A possible pair of new personality traits. A recent hypothesis by Bateson et

\footnotetext{
${ }^{13}$ I am grateful to Mária Herskovits, Cleopatra Ormos and András Szabó for many of the ideas in this paragraph.

${ }^{14}$ The high emotional pressure involved a combination of monetary incentives, peer pressure and social evaluation.
} 
al. (2004) raises the possibility that animals and humans can be divided into two basic phenotypes: the SMALLS who adapted to survival under harsh conditions, and the BIGS who adapted to proliferation using rich resources. ${ }^{15,16}$ Thinking further about the consequences of this division, I would like to put forward the idea that SMALLS are correlated with a 'thrifty', rather introverted psycho-type, while BIGS are correlated with a 'spending', rather extraverted psycho-type. ${ }^{17}$

BIGS do not fear that they will not be able to reciprocate the goods they receive. BIGS are often altruistic, hoping for a later return. BIGS care about possessing as much of the rich resources as possible, explore new possibilities, and make all sorts of alliances to achieve these goals. BIGS expand, explore and make long-range contacts, which are weak links. In contrast, SMALLS try to spend as little as possible. SMALLS minimize their contacts, which are costly to build and maintain. SMALLS withdraw, restrict, and maintain only the safe, long-term contacts, which are strong links.

Bateson et al. (2004) also added that these phenotypes cannot change from one moment to another. For a complete change, one must wait 2 to 3 generations. ${ }^{18}$ Consequently, if Bateson et al. (2004) are right - and their arguments are rather convincing - then we have fairly distinct STRONGLINKER and WEAKLINKER phenotypes, in both animal and human behavior. A few features of the extreme versions of both STRONGLINKER and WEAKLINKER behavior are listed in Table 7.2.

\section{STRONGLINKERS and WEAKLINKERS are equally}

good. I would like to stress that both phenotypes are equally important and valuable. Without either of them, humankind would have been wiped out

\footnotetext{
${ }^{15}$ The SMALL phenotype would correspond to a modified version of the 'spore' and 'dauer' states of more primitive organisms, where these local energy minima in the phenotype landscape are even more widely separated. Formerly, a similar typology was called the thrifty geno- or phenotype, referring to the genetic consequences of the lifestyle change after the excessive hunting period, which made big game extinct approximately 11000 years ago in the Paleolithic (Neel, 1962), or to the effects of poor nutrition in fetal or early postnatal life, resulting in a sensitivity to diabetes as well as obesity, high blood pressure and other elements of the metabolic syndrome (Hales and Barker, 1992).

${ }^{16}$ The names are a bit misleading. The SMALL and the BIG phenotypes are complex traits and cannot be reduced simply to someone's height.

${ }^{17}$ The dichotomy of the SMALL and the BIG phenotypes can be extended even further. The same distinct response pair is a valid description of plants and ecosystems, which usually display two different and widespread evolutionary responses (D'Odorico et al., 2005).

${ }^{18}$ As an indirect proof of this, certain spending habits, e.g., charity donations, decrease in lawful tax-evasion tactics, should show a roughly two-generation lagtime after the general appearance of discretionary income in a population.
} 
by natural selection a very long time ago. STRONGLINKERS are necessary to build the core of our networks. Even more importantly, STRONGLINKERS are the only solution during hard times and stress. WEAKLINKERS are useful in periods of expansion. However, they have to be controlled by STRONGLINKERS, otherwise the network will overspend its resources, and become inefficient, overconnected and unstable.

STRONGLINKERS and WEAKLINKERS are not always different. Bateson et al. (2004) argued that the phenotypes corresponding to STRONGLINKERS and WEAKLINKERS are a kind of inherited 'fate', and may change only after 2 to 3 generations, in which members experience the opposite environment to an earlier one. If our grandparents and parents lived in relative poverty, we may have a winning lottery ticket, but this does not mean that we will transform from a STRONGLINKER to a WEAKLINKER from one day to the next. The following three notes aim to give a more detailed picture of this rather deterministic statement to help the reader to understand it better.

- STRONGLINKERS and WEAKLINKERS both have strong and weak links. Both STRONGLINKERS and WEAKLINKERS represent only trends, which mean that WEAKLINKERS will definitely have a few strong links, and STRONGLINKERS may easily end up building a weak link.

- A mixed strategy wins in uncertainty. As circumstances evolve, we change from STRONGLINKERS to WEAKLINKERS or vice versa. This does not happen like a kind of miracle. After decades of hidden development, the former WEAKLINKER does not suddenly wake up one morning as a STRONGLINKER. A mixed psycho-type may easily occur for generations. Usually a mixed strategy is not a winning strategy. However, the mixed strategy might become a winning strategy in times of uncertainty, when outside signals suggest the development of both psycho-types. As a rather far-removed but suggestive piece of evidence for this behavior, a mixed strategy becomes the winner in dryland plant ecosystems which have been exposed to random interannual fluctuations in precipitation (D'Odorico et al., 2005).

- Social dimensions. The parallel development of STRONGLINKER and WEAKLINKER behavior may appear in different social dimensions. We may behave like a STRONGLINKER at home with our family and manifest a WEAKLINKER character with our friends, lover, and colleagues, or vice versa. ${ }^{19}$

\footnotetext{
${ }^{19}$ I am grateful to András Szabó for these ideas.
} 
Table 7.2. Differences between the STRONGLINKER and WEAKLINKER personality traits. The table lists typical features of extreme STRONGLINKER and WEAKLINKER personality traits for the sake of easy discrimination between the two. In reality, various mixtures of the above extremes are usually present

\begin{tabular}{|c|c|c|}
\hline Feature & STRONGLINKER behavior & WEAKLINKER behavior \\
\hline $\begin{array}{l}\text { Friendship } \\
\text { network }\end{array}$ & $\begin{array}{l}\text { Relies more on family links, usu- } \\
\text { ally has only a few very good } \\
\text { friends, and masters reliable, } \\
\text { life-long contacts }\end{array}$ & $\begin{array}{l}\text { Has friends from a rather broad } \\
\text { circle, including different back- } \\
\text { grounds and lifestyles, but may } \\
\text { lack close friends; new friends } \\
\text { often become more important } \\
\text { than old ones }\end{array}$ \\
\hline $\begin{array}{l}\text { Cognitive and } \\
\text { emotional } \\
\text { hierarchy }\end{array}$ & $\begin{array}{l}\text { Centered on a few ideas and } \\
\text { emotions; change of cognitive } \\
\text { sets is difficult; highly hierarchi- } \\
\text { cal; self-disciplined }\end{array}$ & 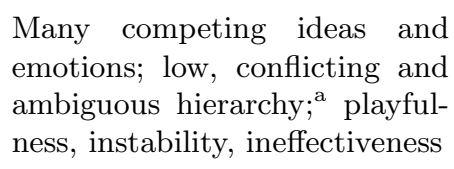 \\
\hline $\begin{array}{l}\text { Internal } \\
\text { images of the } \\
\text { outside world }\end{array}$ & $\begin{array}{l}\text { Rigid, match-seeking usually oc- } \\
\text { curs with pre-set ideas }\end{array}$ & $\begin{array}{l}\text { Flexible, highly adaptive to new } \\
\text { information and environment }\end{array}$ \\
\hline $\begin{array}{l}\text { Cognitive } \\
\text { dimension }\end{array}$ & $\begin{array}{l}\text { Low; can imagine only a few at- } \\
\text { titudes; maximal capacity to un- } \\
\text { derstand interacting people may } \\
\text { be easily saturated }\end{array}$ & $\begin{array}{l}\text { High; can imagine numerous at- } \\
\text { titudes and assess a large num- } \\
\text { ber of interacting people; may } \\
\text { become overcomplicated }\end{array}$ \\
\hline Exploration & $\begin{array}{l}\text { Very efficient; prefers short } \\
\text { range search with very rare } \\
\text { jumps; exponent of Levy flight }{ }^{\text {b }} \\
\text { is big }\end{array}$ & $\begin{array}{l}\text { Inefficient; enjoys longer jumps, } \\
\text { unexpected explorations; the } \\
\text { exponent of the Levy flight is } \\
\text { small; may shift towards ran- } \\
\text { dom walk }\end{array}$ \\
\hline Relaxation & $\begin{array}{l}\text { Disturbed to keep rare energy } \\
\text { packages in the system }\end{array}$ & $\begin{array}{l}\text { Undisturbed to release surplus } \\
\text { energy as fast as possible }\end{array}$ \\
\hline $\begin{array}{l}\text { Ambiguity } \\
\text { tolerance }\end{array}$ & Low & High \\
\hline $\begin{array}{l}\text { Efficiency in } \\
\text { well-defined } \\
\text { tasks }\end{array}$ & High & Low \\
\hline
\end{tabular}


Table 7.2. Cont. Differences between the STRONGLINKER and WEAKLINKER personality traits

\begin{tabular}{|c|c|c|}
\hline Feature & STRONGLINKER behavior & WEAKLINKER behavior \\
\hline $\begin{array}{l}\text { Creative } \\
\text { behavior }\end{array}$ & $\begin{array}{l}\text { High in structured, hierarchical } \\
\text { schemes, like engineering, math- } \\
\text { ematics, religion }\end{array}$ & $\begin{array}{l}\text { High in diffuse structures, like } \\
\text { social sciences, humanities, in- } \\
\text { terdisciplinary research, or art }\end{array}$ \\
\hline $\begin{array}{l}\text { Attitude } \\
\text { towards } \\
\text { resources }\end{array}$ & $\begin{array}{l}\text { Spending-conscious; thrifty; } \\
\text { minimizes excess expenditure }\end{array}$ & $\begin{array}{l}\text { Achievement-conscious; gener- } \\
\text { ous; maximizes income }\end{array}$ \\
\hline $\begin{array}{l}\text { Major } \\
\text { problems }\end{array}$ & $\begin{array}{l}\text { Loneliness, depression, too rigid, } \\
\text { too logical lifestyle, instability } \\
\text { due to absence of weak links }\end{array}$ & $\begin{array}{l}\text { Unfocused life, variable motiva- } \\
\text { tions, lack of endurance, ineffec- } \\
\text { tiveness; instability due to over- } \\
\text { connectedness }\end{array}$ \\
\hline $\begin{array}{l}\text { Network } \\
\text { structure }\end{array}$ & $\begin{array}{l}\text { Scale-free network gets closer to } \\
\text { a star network }\end{array}$ & $\begin{array}{l}\text { Scale-free network gets closer to } \\
\text { a random network }\end{array}$ \\
\hline
\end{tabular}

${ }^{\text {a }}$ I am grateful to Cleopatra Ormos for this idea.

b An efficient search pattern (Levy, 1937) with a scale-free distance distribution (see Sect. 2.2).

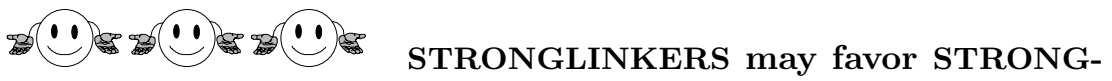
LINKERS while WEAKLINKERS may favor WEAKLINKERS. Pettijohn (1999) has shown that, in stagnant and pessimistic social conditions, American movie actresses with mature facial features have been more popular. However, when social and economic conditions were prosperous and optimistic, actresses with neonate facial features were popular. This preference was not valid for male actors and the preference may be derived from many sources including perceived competence, perceived nurturing abilities, etc. In spite of these restrictions and explanations, let me play with the idea that facial differences also reflect differences in facial symmetry. The neonatal face is more symmetrical than most mature faces. As discussed in Sect. 6.3, increased symmetry reflects lower stress during embryonic development and is probably related to more weak links in cellular networks providing a greater stability. A neonatal face may be more typical of a WEAKLINKER than a STRONGLINKER. During prosperous and optimistic social conditions, society presumably contains a greater number of WEAKLINKERS than STRONGLINKERS. As a grossly simplified explanation for the above findings, during prosperous and optimistic times, the WEAKLINKER social majority selected a WEAKLINKER actress as a preference. In connection with these remarks, it would be very interesting to analyze the results of 


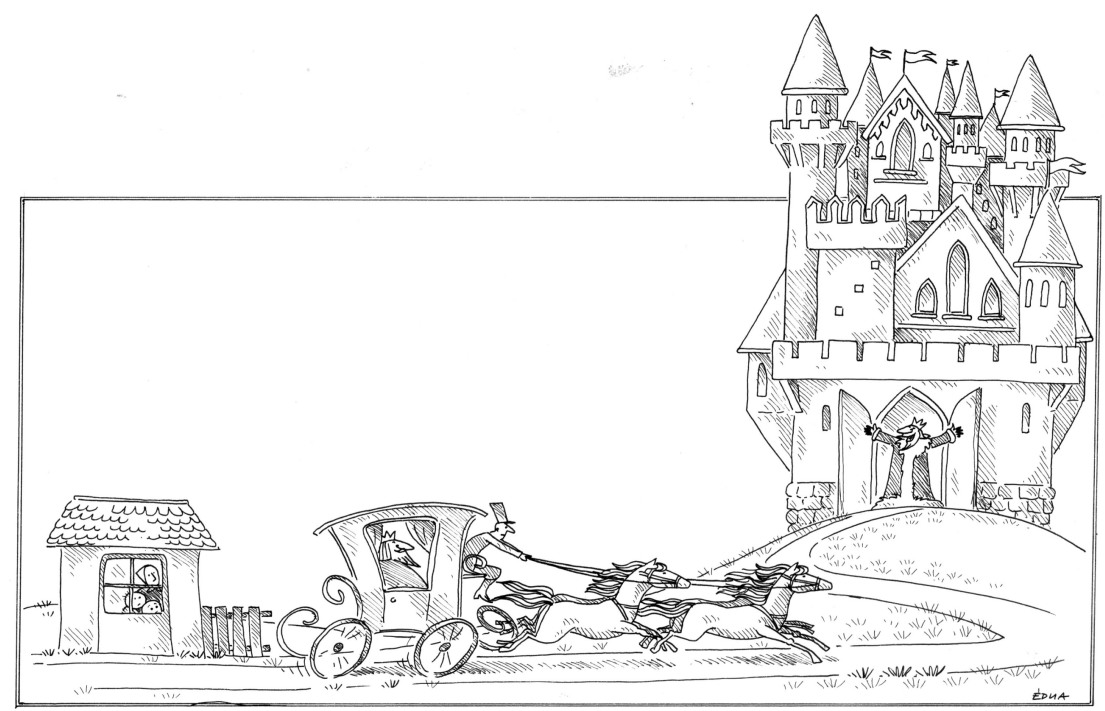

Fig. 7.2. BIGS expand, explore and make long-range contacts which are weak links. SMALLS withdraw, restrict and maintain only the safe, long-lasting contacts, which are strong links

recent US congressional elections (Todorov et al., 2005), where in $70 \%$ of the districts a mature-faced candidate won, as opposed to $30 \%$ of the districts, where a baby-faced candidate was the winner. The election results should correlate with the US population density. The reason for this expectation is that the US population density is the only population measure which correlates with the average height of the current US population (Komlos, 2005). Population density and average height correspond to crowding stress and richness in resources, respectively. Hence, where population density is low, a WEAKLINKER social majority is expected, which may select a WEAKLINKER, baby-faced candidate in the elections. ${ }^{20}$

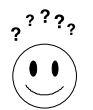

The benefits of linker-symbiosis: Are STRONGLINKERS necessary to protect WEAKLINKERS? I have described the help

\footnotetext{
${ }^{20}$ This expectation may be spoilt if sparse populations have no chance of building social links and become STRONGLINKERS due to their isolation. One might argue that, at the current level of technical development in the USA, this may no longer be a typical situation. However, data collected by Liben-Nowell et al. (2005) showed the extreme importance of geographical distribution on the development of virtual, Internet-based friendship networks, which means that people have a very strong sense of their geographical status and population density even today.
} 
given by low achievers to high achievers in the cellular metabolic net in Sect. 3.3. Low achievers are bad enzymes with a stable structure, whilst high achievers are good enzymes with a labile structure (Shoichet et al., 1995). Perturbations might remain longer with the low achievers. In this way low achievers may protect their more vulnerable, high-achieving counterparts, thus facilitating the record-breaking enzyme activities of the high achievers. A rather loose analogy with a network two levels higher, the social net, raises the following question: are STRONGLINKERS necessary to protect WEAKLINKERS in society? Is trouble dissipation by STRONGLINKERS a mandatory 'trouble sink' in society to keep the vital bridges of the sensitive WEAKLINKERS alive? Can one say that STRONGLINKERS build local circles, helping and ensuring the local dissipation of trouble, while WEAKLINKERS provide global coupling to ensure the emergent properties of the social network as a whole? Do WEAKLINKERS build a rich club, where 'club members' have a large number of connections? Where can we position the rich clubs of WEAKLINKERS in society? Are WEAKLINKERS mostly members of the middle, or upper-middle class? Do the really rich become STRONGLINKERS again, and withdraw to a VIP club building only a small number of strong links, and directing a few influential WEAKLINKERS to go out and cause trouble (Masuda and Konno, 2005)? It would be interesting to examine assortative social networks from this point of view.

It is a basic rule of psychotherapy that the psychoanalyst cannot establish strong personal contact with the patient. "I have not participated in any psychotherapy yet, but I cannot believe that you are right. How can any therapy be successful without a strong contact?" Intensity and strength are not the same, Spite. Psychotherapeutic contact is a very intensive contact, but it is both purpose-oriented and limited to the sessions. This is why it remains a weak link between the two multidimensional personalities. The task-oriented approach is an element of the success here. Besides the ethical norm which prevents the therapist from exploiting the patient, many examples show that a sufficient distance is needed to give real psychological help (Degenne and Forse, 1999; Freud, 1915; Kawachi and Berkman, 2001; Veiel, 1993). Weak links seems to help our stabilization better than strong bonds.

Weak links stabilize partnerships. It is rather commonplace but still worth noting here that long-term partnerships and the health of their participants are stabilized by the weak links of affection rather than the strong links of burning love. As a modern form of this, care can be ex- 
pressed more by frequent SMS messages than by a limited number of lengthy phone calls. Frequent SMS messages here correspond to multiple weak links as opposed to lengthy calls corresponding to occasional strong links. ${ }^{21}$

Social networks may influence the psyche in a number of ways. However, here the interpersonal links are much more important than the intrapersonal network structure. I will therefore list the remaining examples of these interactions in Sect. 10.3, under the heading of social capital.

Leaving our body (do not worry, I neither drank too much nor invite you to come with me for a splendid session of levitation; we just finished our third trip into Netland), let me summarize what has been gathered together here. We have gleaned quite a bit of evidence according to which various networks in our body, such as the immunological networks, the blood flow system, the muscle net, the neuro-glial network, and our psyche, all involve a large number of weak links. Moreover, this part of Netland is found to be full of smoking guns. In all these networks, there were quite suggestive hints that they might be stabilized by weak links. However, most of the formal proofs are still lacking. Perhaps it is time to start work!

${ }^{21}$ I am grateful to István Kovács for this idea. 